Since $u_{r}=U\left(T_{r}^{-1}\right)$ it is clear that $H=H_{1}$. Since $T_{r}=r T_{1}$, it follows that $T_{r}^{-1}(z)=T_{1}^{-1}(z / r)$ so that $H_{r}(z)=H_{1}(z / r)$. Taking the limit as $r$, $0<r<1$, approaches one, one has

as required.

$$
H(0)=\lim _{r \rightarrow 1} H_{r}(0) \leqq \ln 2+\ln 2=\ln 4,
$$

\title{
BIBLIOGRAPHY
}

1. W. C. Fox, $A$ new inequality for the Green's function, Proc. Amer. Math. Soc. vol. 10 (1959) pp. 562-569.

NORTHWESTERN UNIVERSITY

\section{SOME DIFFERENTIAL GEOMETRIC PROPERTIES OF SUBMANIFOLDS OF EUCLIDEAN SPACES}

\author{
ROBERT E. STONG
}

1. Introduction. The translation theorem of Hopf and Voss [3] has been generalized by Hsiung [4] and Voss [7] to hypersurfaces, by Hsü [6] to other elementary transformations, and these results were obtained for hypersurfaces by Aeppli [1]. In the first sections of this paper, these results will be generalized to $n$-dimensional manifolds in $(n+m)$-dimensional Euclidean space. In the final section, a condition will be given under which a submanifold of Euclidean space is a submanifold of a hypersphere, extending a result of Hsiung [5].

All manifolds mentioned will be assumed to be compact, connected, orientable, $n$-dimensional $(n \geqq 2)$ manifolds with closed boundaries (empty or of dimension $n-1)$ differentiably imbedded in an $(n+m)$ dimensional Euclidean space $E^{n+m}(m \geqq 1)$. The notation adopted will be essentially that of Hsiung [4]. The following conventions will be adopted for indices:

$$
\begin{aligned}
\alpha, \beta, \cdots & =1, \cdots, n+m, \\
\lambda, \mu, \cdots & =1, \cdots, n+m-1, \\
a, b, \cdots & =1, \cdots, m, \\
i, j, \cdots & =1, \cdots, n, \\
r, s, \cdots & =0, \cdots, n .
\end{aligned}
$$

Considerable use will be made of a vector product like that defined by Hsiung [5]. Namely, if $I_{1}, \cdots, I_{n+m}$ are a fixed frame of mutually

Received by the editors July 11, 1960. 
orthogonal unit vectors and $A_{\lambda}=A_{\lambda}^{\alpha} I_{\alpha}$ are any $(n+m-1)$ vectors,

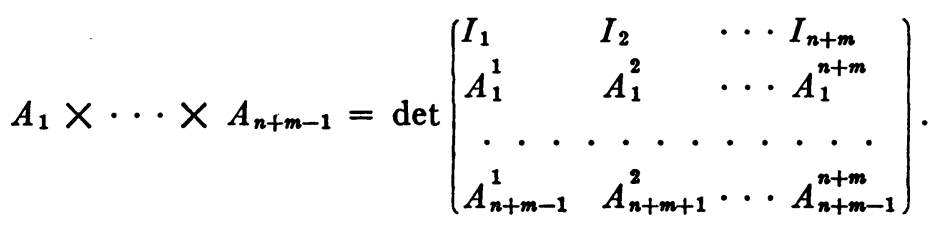

Further, the exterior convention for multiplication of differentials will be observed in vector products.

If $N_{1}, \cdots, N_{m}$ is a frame of mutually orthogonal unit vector fields normal to a manifold $V^{n}$ (locally) with

$$
X_{11} \cdot X_{12} \times \cdots \times X_{1 n} \times N_{1} \times \cdots \times N_{m}>0
$$

where ${ }_{1 i}$ denotes partial differentiation with respect to the $i$ th local coordinate, then one has

$\frac{n-r}{d X \times \cdots \times d X} \times \overbrace{d N_{a} \times \cdots \times d N_{a}}^{r} \times N_{1} \times \cdots \times \hat{N}_{a} \times \cdots \times N_{m}$
$=(-1)^{n-r+a-1} n ! M_{r}\left(N_{a}\right) N_{a} d A$

with ^ denoting that the corresponding factor is omitted, and $M_{r}\left(N_{a}\right)$ denoting the $r$ th mean curvature of $V^{n}$ determined by the normal field $N_{a}$.

2. Translation theorem. In this section, the following theorem will be proved.

THEOREM. Let $V^{n}, V^{* n} \subset E^{n+m}$ with boundaries $V^{n-1}, V^{* n-1}$, and let $f: V^{n} \rightarrow V^{* n}$ be an orientation preserving diffeomorphism such that the line segments joining corresponding points are always parallel to a fixed direction e.

Let $S$ be the set of points of $V^{n}$ at which the vector e does not lie in the tangent space, and let $N_{1}$ denote the unit vector field defined on $S$ which lies in the linear space spanned by the tangent space of $V^{n}$ and the vector $e$, is orthogonal to the tangent space, and for which $e \cdot N_{1}>0$.

Suppose further that $S$ is dense in $V^{n}$ and $S \cap V^{n-1}$ is dense in $V^{n-1}$, that on $V^{n-1}, V^{* n-1}$ one has $N_{1}^{*}=N_{1}$ and that at corresponding points $M_{1}^{*}\left(N_{1}^{*}\right)=M_{1}\left(N_{1}\right)$. Then $f$ is a translation.

Proof. Let $X^{*}=X+w e$. On $S, \Gamma=e-\left(e \cdot X_{\mid i}\right) g^{i j} X_{1 j} \neq 0$ and from $X_{1 i}^{*}=X_{1 i}+w_{\mid i} e$, the linear spans $\left[X_{\mid 1}^{*}, \cdots, X_{\mid w}^{*} e\right]$ and $\left[X_{\mid 1}, \cdots, X_{\mid n}, e\right]$ are equal, so that on $f(S), \Gamma^{*}=e-\left(e \cdot X_{\mid i}^{*}\right) g^{* i j} X_{\mid j}^{*}$ $\neq 0$. Then $N_{1}=\Gamma /|\Gamma|, N_{1}^{*}=\Gamma^{*} /\left|\Gamma^{*}\right|$ are the corresponding unit vectors. Choose $N_{2}, \cdots, N_{m}$ normal to $\left[X_{\mid 1}, \cdots, X_{\mid n}, e\right]$ (locally) forming a frame of mutually orthogonal unit vectors with 


$$
X_{11} \cdot X_{12} \times \cdots \times X_{1 n} \times N_{1} \times \cdots \times N_{m}>0 .
$$

(If $m=1$, use this to determine the orientation of $V^{n}$.)

Let $\mathfrak{A}=w e \cdot N_{1}^{*} \times d X \times \cdots \times d X \times N_{2} \times \cdots \times N_{m}=w e \cdot N_{1}^{*}$ $\times d X^{*} \times \cdots \times d X^{*} \times N_{2} \times \cdots \times N_{m}$. Then

$$
\begin{aligned}
d \mathfrak{A}= & w e \cdot d X^{*} \times \cdots \times d X^{*} \times d N_{1}^{*} \times N_{2} \times \cdots \times N_{m}-N_{1}^{*} \\
& \cdot(d w) e \times d X^{*} \times \cdots \times d X^{*} \times N_{2} \times \cdots \times N_{m} \\
& +\sum_{a>1}(-1)^{n-a} w e \\
& \cdot d X^{*} \times \cdots \times d X^{*} \times d N_{a} \times N_{1}^{*} \times \cdots \times \hat{N}_{a} \times \cdots \times N_{m} .
\end{aligned}
$$

Since $X_{\mid 1}^{*} \cdot X_{\mid 2}^{*} \times \cdots \times X_{\mid n}^{*} \times N_{1}^{*} \times N_{2} \times \cdots \times N_{m}>0$, $(-1)^{n} n ! N_{1}^{*} d A^{*}=d X^{*} \times \cdots \times d X^{*} \times N_{2} \times \cdots \times N_{m}$,

$$
\begin{aligned}
= & d X \times \cdots \times d X \times N_{2} \times \cdots \times N_{m} \\
& +n(d w) e \times d X \times \cdots \times d X \times N_{2} \times \cdots \times N_{m}, \\
= & (-1)^{n} n ! N_{1} d A+n(d w) e \times d X \times \cdots \times d X \times N_{2} \times \cdots \times N_{m} .
\end{aligned}
$$

For $a>1, d X^{*} \times \cdots \times d X^{*} \times d N_{a} \times N_{1}^{*} \times \cdots \times N_{a} \times \cdots \times N_{m}$ has the direction of $N_{a}$, and

$$
w e \cdot d X^{*} \times \cdots \times d X^{*} \times d N_{a} \times N_{1}^{*} \times \cdots \times N_{a} \times \cdots \times N_{m}=0 \text {. }
$$

Thus

$$
d \mathfrak{A}=(-1)^{n-1}(n-1) !\left(d A^{*}-N_{1}^{*} \cdot N_{1} d A\right)+(-1)^{n-1} n ! M_{1}^{*}\left(N_{1}^{*}\right) w e \cdot N_{1} d A .
$$

Let $\mathfrak{B}=w e \cdot N_{1} \times d X \times \cdots \times d X \times N_{2} \times \cdots \times N_{m}$. Then

$$
\begin{aligned}
d \mathscr{S}= & w e \cdot d X \times \cdots \times d X \times d N_{1} \times N_{2} \times \cdots \times N_{m}-N_{1} \\
& \cdot(d w) e \times d X \times \cdots \times d X \times N_{2} \times \cdots \times N_{m} \\
& +\sum_{a>1}(-1)^{n-a} w e \\
& \cdot d X \times \cdots \times d X \times d N_{a} \times N_{1} \times \cdots \times N_{a} \times \cdots \times N_{m}, \\
= & (-1)^{n-1}(n-1) !\left(N_{1} \cdot N_{1}^{*} d A^{*}-d A\right)+(-1)^{n-1} n ! M_{1}\left(N_{1}\right) w e \\
& \cdot N_{1} d A .
\end{aligned}
$$

Extending to $V^{n}$ by continuity,

$$
\begin{aligned}
\int_{V^{n}}\left(1-N_{1}^{*} \cdot N_{1}\right)\left(d A+d A^{*}\right) & =\left\{(-1)^{n-1} /(n-1) !\right\} \int_{V^{n-1}}(\mathfrak{A}-\mathfrak{B}), \\
& =0 .
\end{aligned}
$$

Since $\left(1-N_{1}^{*} \cdot N_{1}\right) \geqq 0$ and $f$ preserves orientation, $N_{1}^{*}=N_{1}$ almost everywhere on $S$. At such points, 


$$
0=X_{1 i}^{*} \cdot N_{1}^{*}=X_{1 i}^{*} \cdot N_{1}=w_{1 i} e \cdot N_{1}
$$

and since $e \cdot N_{1} \neq 0, w_{\mid i}=0$. By continuity, $w_{1 i}=0$ on $V^{n}$ and so $w=$ constant.

\section{Similarity theorem.}

Theorem. Let $V^{n}, V^{*_{n}} \subset E^{n+m}$ with boundaries $V^{n-1}, V^{*_{n-1}}$ and let $f: V^{n} \rightarrow V^{*_{n}}$ be a diffeomorphism such that at corresponding points

$$
X^{*}=k X \text {. }
$$

Let $S$ denote the set of points of $V^{n}$ at which the position vector does not lie in the tangent space, and let $N_{1}$ be the unit vector field defined on $S$ which lies in the linear space spanned by the tangent space of $V^{n}$ and the position vector, which is orthogonal to the tangent space, and for which $X \cdot N_{1}>0$.

Then if $S$ is dense in $V^{n}$ and $S \cap V^{n-1}$ is dense in $V^{n-1}$, if on $V^{n-1}$ and $V^{* n-1}$ one has $N_{1}^{*}=N_{1}\left(\right.$ or $\left.N_{1}^{*}=-N_{1}\right)$, and if $k M_{1}^{*}\left(N_{1}^{*}\right)-M_{1}\left(N_{1}\right)=0$ (or $\left.k M_{1}^{*}\left(N_{1}^{*}\right)+M_{1}\left(N_{1}\right)=0\right)$ on $S$, then $f$ is a homotheticity.

Proof. Let $T$ denote the intersection of $S$ and the set of points of $V^{n}$ at which $k \neq 0, \infty$. Since $f$ is a homeomorphism, $S$ and $T$ differ by at most two points.

From $X_{\mid i}^{*}=k X_{\mid i}+k_{\mid i} X$, one has $\left[X_{\mid 1}^{*}, \cdots, X_{\mid n}^{*}, X^{*}\right]$ $\subset\left[X_{\mid 1}, \cdots, X_{\mid n}, X\right]$. Reversing the roles gives equality, so that on $T, \Lambda=X-\left(X \cdot X_{\mid i}\right) g^{i j} X_{\mid j} \neq 0$ implies $\Lambda^{*} \neq 0$. Then $N_{1}=\Lambda /|\Lambda|$, and choose $N_{2}, \cdots, N_{m}$ normal to $\left[X_{\mid 1}, \cdots, X_{\mid n}, X\right]$ (locally) forming a frame of mutually orthogonal unit vectors, with

$$
X_{11} \cdot X_{12} \times \cdots \times X_{1 n} \times N_{1} \times \cdots \times N_{m}>0 .
$$

(If $m=1$, this convention is used to determine the orientation of $V^{n}$ ).

Let $\mathfrak{A}=X \cdot N_{1} \times d X \times \cdots \times d X \times N_{2} \times \cdots \times N_{m}$. Then

$$
\begin{aligned}
d \mathfrak{A}= & X \cdot d X \times \cdots \times d X \times d N_{1} \times N_{2} \times \cdots \times N_{m}-N_{1} \\
& \cdot d X \times \cdots \times d X \times N_{2} \times \cdots \times N_{m} \\
& +\sum_{a>1}(-1)^{n-a} X \\
& \cdot d X \times \cdots \times d X \times d N_{a} \times N_{1} \times \cdots \times N_{a} \times \cdots \times N_{m},
\end{aligned}
$$

and since $X \cdot N_{a}=0$ for $a>1$, this becomes

$$
d \mathfrak{A}=(-1)^{n+1} n ! d A+(-1)^{n+1} n ! M_{1}\left(N_{1}\right) p_{1} d A
$$

where $p_{1}=X \cdot N_{1}$.

Let $\mathfrak{B}=X \cdot N_{1}^{*} \times d X \times \cdots \times d X \times N_{2} \times \cdots \times N_{m}$. Then 


$$
\begin{aligned}
& d \mathfrak{B}=X \cdot d X \times \cdots \times d X \times d N_{1}^{*} \times N_{2} \times \cdots \times N_{m} \\
& -N_{1}^{*} \cdot d X \times \cdots \times d X \times N_{2} \times \cdots \times N_{m} \\
& +\sum_{a>1}(-1)^{n-a} X \cdot d X \times \cdots \times d X \times d N_{a} \times N_{1}^{*} \times \cdots \\
& \times \hat{N}_{a} \times \cdots \times N_{m} \\
& =k^{-n} X^{*} \cdot d X^{*} \times \cdots \times d X^{*} \times d N_{1}^{*} \times N_{2} \times \cdots \times N_{m} \\
& -N_{1}^{*} \cdot d X \times \cdots \times d X \times N_{2} \times \cdots \times N_{m} \\
& +\sum_{a>1}(-1)^{n-a} k^{-n} X^{*} \cdot d X^{*} \times \cdots \times d X^{*} \times d N_{a} \times N_{1}^{*} \times \cdots \\
& \times \hat{N}_{a} \times \cdots \times N_{m} \\
& =k^{-n} \xi(-1)^{n-1} n ! M_{1}^{*}\left(N_{1}^{*}\right) X^{*} \cdot N_{1}^{*} d A^{*}+(-1)^{n+1} n ! N_{1}^{*} \cdot N_{1} d A,
\end{aligned}
$$

where $\xi=$ sign of $\left(X_{11}^{*} \cdot X_{\mid 2}^{*} \times \cdots \times X_{\mid n}^{*} \times N_{1}^{*} \times N_{2} \times \cdots \times N_{m}\right)$.

From

$$
\begin{aligned}
\xi(-1)^{n} n ! N_{1}^{*} d A^{*} & =d X^{*} \times \cdots \times d X^{*} \times N_{2} \times \cdots \times N_{m} \\
& =k^{n} d X \times \cdots \times d X \times N_{2} \times \cdots \times N_{m} \\
& +n k^{n-1}(X d k) \times d X \times \cdots \times d X \times N_{2} \times \cdots \times N_{m},
\end{aligned}
$$

one has $\xi(-1)^{n} n ! X^{*} \cdot N_{1}^{*} d A^{*}=(-1)^{n} k^{n+1} n ! X \cdot N_{1} d A$, so

$$
d \mathfrak{O}=(-1)^{n+1} n ! N_{1}^{*} \cdot N_{1} d A+(-1)^{n+1} n ! k M_{1}^{*}\left(N_{1}^{*}\right) p_{1} d A .
$$

Thus (for the two cases),

$$
\begin{aligned}
\int_{V^{n}}\left(1 \mp N_{1}^{*} \cdot N_{1}\right) d A & =\left\{(-1)^{n+1} / n !\right\} \int_{V^{n-1}}(\mathfrak{A} \mp \mathfrak{B}) \\
& =0 .
\end{aligned}
$$

Since $1 \mp N_{1}^{*} \cdot N_{1} \geqq 0, N_{1}^{*}= \pm N_{1}$ almost everywhere on $T$, and at these points

$$
0=X_{\mid i}^{*} \cdot N_{1}^{*}= \pm X_{1 i}^{*} \cdot N_{1}= \pm k_{\mid i} X \cdot N_{1}= \pm k_{\mid i} p_{1} .
$$

Thus $k_{1 i}=0$ almost everywhere. By continuity, $k_{1 i}$ vanishes throughout $V^{n}$, so $k$ is a constant.

4. Characterization of manifolds in hyperspheres. A manifold in $E^{n+m}$ will be said to be star shaped with respect to the origin if at each point the position vector does not belong to the tangent space at that point. As in $\S 3, N_{1}$ will denote the unit vector field on $V^{n}$ which lies in the linear space spanned by the tangent space and the position vector, which is orthogonal to the tangent space and for which $X \cdot N_{1}>0$. Then 


$$
X=p_{1} N_{1}+\left(X \cdot X_{\mid i}\right) g^{i j} X_{\mid j}
$$

where $p_{1}=X \cdot N_{1}>0$.

$\Delta$ will denote the Laplacian: $\Delta \phi=\phi_{; i j} g^{i j}$, where semicolons denote covariant differentiation with respect to the Christoffel symbols formed from $g_{i j}$. One finds by straightforward calculation that:

Lemma 1. For any submanifold $V^{n}$ of $E^{n+m}$, the mean curvature normal is $1 / n$ times the Laplacian of the position vector.

Lemma 2. On a manifold which is star shaped with respect to the origin, one has $\Delta(X \cdot X)=2 n\left(1+p_{1} M_{1}\left(N_{1}\right)\right)$.

Proof.

$$
\begin{aligned}
\Delta(X \cdot X) & =2 X \cdot \Delta X+2 X_{1 i} \cdot X_{1 j} g^{i j} \\
& =2\left(p_{1} N_{1}+\left(X \cdot X_{1 i}\right) g^{i j} X_{\mid j}\right) \cdot \Delta X+2 g_{i j} g^{i j} \\
& =2 n p_{1} M_{1}\left(N_{1}\right)+2 n,
\end{aligned}
$$

for by Lemma $1,(1 / n) \Delta X \cdot N_{1}=M_{1}\left(N_{1}\right)$ and $\Delta X \cdot X_{\mid i}=0$.

LEMMa 3. On a manifold which is star shaped with respect to the origin one has

$$
\Delta\left(|X|^{-x+2}\right)=(2-\chi)|X|^{-x}\left\{n M_{1}\left(N_{1}\right) p_{1}+n-\chi\left(1-p_{1}^{2} /|X|^{2}\right)\right\}
$$

and

$$
\Delta(\log |X|)=|X|^{-2}\left\{n M_{1}\left(N_{1}\right) p_{1}+n-2\left(1-p_{1}^{2} /|X|^{2}\right)\right\} .
$$

Proof. From

$$
\begin{aligned}
\Delta\left(\phi^{\lambda}\right) & =\lambda \phi^{\lambda-1} \Delta \phi+\lambda(\lambda-1) \phi^{\lambda-2} \phi_{1 i} \phi_{1 j} g^{i j}, \\
2 n p_{1} M_{1}\left(N_{1}\right)+2 n & =2|X| \Delta|X|+2|X|_{1 i}|X|_{1 j} g^{i j}
\end{aligned}
$$

and since $X \cdot X_{\mid i}=(1 / 2)(X \cdot X)_{\mid i}=(1 / 2)\left(|X|^{2}\right)_{\mid i}=|X||X|_{\mid i}$ yields

$$
\begin{aligned}
p_{1}^{2} & =\left\{X-\left(X \cdot X_{\mid i}\right) g^{i j} X_{\mid j}\right\} \cdot\left\{X-\left(X \cdot X_{\mid h}\right) g^{h k} X_{\mid k}\right\} \\
& =X \cdot X-\left(X \cdot X_{\mid i}\right) g^{i j}\left(X \cdot X_{\mid j}\right) \\
& =|X|^{2}\left(1-g^{i j}|X|_{\mid i}|X|_{\mid j}\right),
\end{aligned}
$$

one has the first result. Then

$$
\begin{aligned}
|X|^{-2}\left\{n M_{1}\left(N_{1}\right) p_{1}+n-2\left(1-p_{1}^{2} /|X|^{2}\right)\right\} & \\
& =\lim _{x \rightarrow 2} \Delta\left(|X|^{-x+2}\right) /-x+2,
\end{aligned}
$$

and applying L'Hospital's rule gives the second result. 
THEOREM. Let $V^{n} \subset E^{n+m}$ have empty boundary and be star shaped with respect to the origin. If $M_{1}\left(N_{1}\right) \leqq-1 /|X|$ (or $\left.M_{1}\left(N_{1}\right) \geqq-1 / p_{1}\right)$ throughout $V^{n}$, then $V^{n}$ is a submanifold of a hypersphere with center at the origin.

Proof. If $M_{1}\left(N_{1}\right) \leqq-1 /|X|, \quad M_{1}\left(N_{1}\right) p_{1} \leqq-p_{1} /|X| \leqq-p_{1}^{2} /|X|^{2}$ since $0<p_{1} \leqq|X|$. By Lemma 3,

$$
n|X|^{-n}\left\{M_{1}\left(N_{1}\right) p_{1}+p_{1}^{2} /|X|^{2}\right\}=\left\{\begin{array}{cc}
\Delta\left(|X|^{-n+2}\right) / 2-n & \text { if } n \neq 2, \\
\Delta(\log |X|) & \text { if } n=2,
\end{array}\right.
$$

so $\Delta\left(|X|^{-n+2}\right) \geqq 0$ (or $\Delta(\log |X|) \leqq 0$ ). By Bochner's Lemma (see Yano and Bochner [8]), this implies $|X|=$ constant.

If $M_{1}\left(N_{1}\right) \geqq-1 / p_{1}$, one has from Lemma 2 that $\Delta(X \cdot X) \geqq 0$ and $X \cdot X$ is constant.

Note. These results may be obtained as in $\$ 3$, by letting $V^{n} \rightarrow S^{n+m-1}: X \rightarrow X^{\prime}=X /|X|$. Then if $D=X^{\prime} \cdot N_{1} \times d X^{\prime} \times \cdots \times d X^{\prime}$ $\times N_{2} \times \cdots \times N_{m}, \mathfrak{A}=X \cdot N_{1} \times d X \times \cdots \times d X \times N_{2} \times \cdots$ $\times N_{m}$, one has

$$
\begin{aligned}
\int_{V^{n}}|X|^{-n}\left\{M_{1}\left(N_{1}\right) p_{1}+p_{1}^{2} /|X|^{2}\right\} d A & =\left\{(-1)^{n+1} / n !\right\} \int_{V^{n-1}} D \\
\int_{V^{n}}\left\{M_{1}\left(N_{1}\right) p_{1}+1\right\} d A & =\left\{(-1)^{n+1} / n !\right\} \int_{V^{n-1}} \mathfrak{A} .
\end{aligned}
$$

Combining these with the assumptions of the theorem, one has $p_{1}=|X|$ which implies $|X|$ is constant.

\section{REFERENCES}

1. A. Aeppli, Einige Ähnlichkeits- und Symmetriesätze für differenzierbare Flächen im Raum, Comment. Math. Helv. vol. 33 (1959) pp. 174-195.

2. L. P. Eisenhart, Riemannian geometry, Princeton University Press, 1926.

3. H. Hopf and K. Voss, Ein Satz aus der Flächentheorie im Grossen, Arch. Math. vol. 3 (1952) pp. 187-192.

4. C. C. Hsiung, Some global theorems on hypersurfaces, Canad. J. Math. vol. 9 (1957) pp. 5-14.

5. - Some integral formulas for closed hypersurfaces, Math. Scand. vol. 2 (1954) pp. 286-294.

6. C. S. Hsü, Characterization of some elementary transformations, Proc. Amer. Math. Soc. vol. 10 (1959) pp. 324-328.

7. K. Voss, Einige differentialgeometrische Kongruenzsätze für geschlossene Flächen und Hyperflächen, Math. Ann. vol. 131 (1956) pp. 180-218.

8. K. Yano and S. Bochner, Curvature and Betti numbers, Princeton University Press, 1953.

University of Chicago 